\title{
Efectos de la actividad física sobre las funciones ejecutivas en una muestra de adolescentes
}

\author{
Inmaculada Martín-Martínez ${ }^{1 *}$, Luis J. Chirosa-Ríos ${ }^{1}$, Rafael E. Reigal-Garrido ${ }^{1}$, Antonio Hernández-Mendo ${ }^{2}$, \\ Rocío Juárez-Ruiz-de-Mier ${ }^{3}$ y Rafael Guisado-Barrilao ${ }^{1}$ \\ 1 Universidad de Granada (España). \\ 2 Universidad de Málaga (España). \\ 2 Hospital Xanit Internacional, Benalmádena (España).
}

\begin{abstract}
Resumen: El propósito de este trabajo fue analizar los efectos de un programa de actividad física, con una duración de 8 semanas y basado en juegos reducidos, sobre diversas variables cognitivas vinculadas al constructo funciones ejecutivas como memoria de trabajo, inhibición y flexibilidad cognitiva. Los participantes fueron 54 adolescentes, físicamente inactivos, de la ciudad de Priego (Córdoba, España), con edades entre los 15 y 16 años $(M=15.35 ; D T=.48)$. Se empleó un diseño pre-post con dos grupos aleatorizados, control y experimental. Los instrumentos utilizados para evaluar las habilidades cognitivas fueron el test Dígitos y el test Letras y Números, de la Escala de Inteligencia de Wechsler para Niños (WISC-IV), el Trail Making Test y el Test Stroop. Se realizó un análisis de la varianza mixta 2x2 para determinar los efectos del tratamiento. Los resultados indicaron efectos positivos del programa de actividad física sobre la memoria de trabajo y la flexibilidad cognitiva, aunque con un tamaño del efecto bajo. Se propone incrementar el tiempo de intervención y aplicar diferentes programas para observar el impacto del tipo de actividad en futuras investigaciones.

Palabras clave: actividad física; adolescencia; cognitivo; juegos reducidos.
\end{abstract}

\section{Introducción}

Los beneficios que la práctica de actividad física tiene sobre los seres humanos se ha puesto de manifiesto en numerosas investigaciones (Gómez-Cabello, Ara, González-Agüero, Casajús y Vicente-Rodríguez, 2012; Mota, Santos, Silva, Aires, Martins y Vale, 2012; Padilla-Moledo et al., 2012). En concreto, la relación entre la práctica física y el funcionamiento cognitivo, aunque ha sido objeto de interés desde hace muchos años, sólo desde hace varias décadas se está evaluando de manera sistemática. Entre otros motivos, el avance de la tecnología y el perfeccionamiento de técnicas como la neuroimagen contribuyen a dar respuesta cuestiones que han sido difíciles de resolver anteriormente (Chaddock et al., 2010; Hillman, Erickson y Kramer, 2008). Aunque la mayoría de los estudios se han ocupado de población adulta y avanzada edad, existen aquellos que han centrado su interés en niños y adolescentes. Además, este fenómeno ha sido identificado tanto tras la realización de una sesión de ejercicio físico como después de un programa estructurado en el tiempo, siendo el ejercicio aeróbico el que ha demostrado mayor efectividad (Chaddock, Hillman, Pontifex, Jonhson, Raine y Kramer, 2011; Kempermann et al., 2010; O’Leary, Pontifex, Scudder, Brown y Hillman, 2011).

* Dirección para correspondencia [Correspondence address]

Inmaculada Martín Martínez. Grupo Investigación CTS-642, Departamento de Educación Física y Deportiva, Facultad de Ciencias del Deporte, Universidad de Granada, Granada, España. Carretera de Alfacar s/n, 18011, Granda (España). E-mail: inmartintinez@hotmail.com
Title: Effects of physical activity on executive function in a sample of adolescents.

Abstract: The aim of this study was to analyse the effects of a physical ac tivity program, eight-week and based on small sided games, on some variables associated to executive functions construct such as working memory, inhibition and cognitive flexibility. The participants in the study were 54 adolescents, who were physically inactive, from the city of Priego (Córdo$\mathrm{ba}$, Spain), whose age ranged between 15 and 16 years old $(M=15.35 ; S D=$ .48). The study was based on a quasi-experimental pre-post design with two randomized groups, control and experimental. The instruments used to evaluate the cognitive skills were Digits and Letters and Numbers tests of the Wechsler Intelligence Scale for Children (WISC-IV), the Trail Making Test (A and B) and the Stroop Test. It was realized an analysis of the mixed variance $2 \times 2$ to determine the effects of the treatment. Results showed positive effects of the physical activity program on working memory and cognitive flexibility, although the effect size was weak. It is proposed to increase the time of intervention and apply different treatments to observe the impact of type of activity for future research.

Key words: physical activity; adolescence; cognitive; small sided games.

La cognición es un término general referido a un conjunto de procesos mentales que el ser humano es capaz de llevar a cabo. En este espectro, las funciones ejecutivas es un constructo empleado para aglutinar a una serie de capacidades cognitivas implicadas en el control del pensamiento y la conducta (Zelazo y Carlson, 2012). Entre otras habilidades, permiten organizar y planificar una tarea, seleccionar adecuadamente unos objetivos, iniciar un plan de acción y mantenerlo mentalmente, ser flexible en las estrategias para alcanzar una meta o inhibir estímulos irrelevantes (Banich, 2009; Shallice, 1994; Soprano, 2003). Además, son esenciales para adaptarse al medio y tener un funcionamiento social adecuado. Asimismo, en ámbitos específicos como el deportivo, están implicadas en el éxito de numerosas tareas que hay que resolver durante la práctica física (Davidson, Amso, Anderson y Diamond, 2006; Hillman, Snook y Jerome, 2003; Lezak, 2004).

Diversos modelos han pretendido contextualizar las bases teóricas, funcionales y neuroanatómicas de las funciones ejecutivas. De esta forma existen aquellos que las consideran un constructo único y otros que sugieren una estructura disociada, aunque pudieran sus componentes relacionarse entre sí (Burgess et al., 2006; Stelzer, Mazzoni y Cervigni, 2014). Desde esta última perspectiva, Diamond (2006) propuso que la memoria de trabajo, el control inhibitorio y la flexibilidad cognitiva eran procesos constituyentes de las funciones ejecutivas aunque con tendencias evolutivas particulares. De igual modo, señaló que la capacidad que poseían estos elementos para coordinarse tenía unos periodos 
más sensibles que otros a lo largo del desarrollo de la propia persona.

Diversos trabajos han centrado su interés en relacionar la actividad física, tanto aguda como prolongada, con las funciones ejecutivas en niños y adolescentes, siendo recogidos en diferentes revisiones (Best, 2010; Tomporowski, Davis, Miller y Naglieri, 2008; Tomporowski, Lambourne y Okumura, 2011). En ellas, uno de los aspectos reseñables es la existencia de mayor cuerpo de conocimiento en población infantil y preadolescente que en edades más avanzadas. Como ejemplo, en un estudio realizado por Davis et al. (2011) sobre 171 niños con sobrepeso $\left(\mathrm{IMC}=26 \pm 4.6 \mathrm{~kg} / \mathrm{m}^{2}\right)$ entre 7 y 11 años, encontraron que tras un programa de 13 semanas de ejercicio aeróbico, hubo un beneficio sobre la capacidad de planificación. Otro trabajo realizado por Hillman, Pontifex, Raine, Castelli, Hall y Kramer (2009) con un grupo de 20 niños preadolescentes con una media de edad de 9.5 años, señaló el efecto agudo que tuvo un ejercicio aeróbico en tapiz rodante, que consistía en caminar durante 20 minutos al $60 \%$ de la frecuencia cardíaca máxima, sobre el control inhibitorio. En otro estudio de Kubesch et al. (2009), con 81 adolescentes alemanes entre 13 y 14 años, observaron los efectos agudos positivos de 30 minutos de ejercicio aeróbico sobre la inhibición y la memoria de trabajo.

En esta línea, aunque con diferente enfoque, otros trabajos se han centrado en valorar aspectos de la condición física y su relación con las funciones ejecutivas. Buck, Hillman y Castelli (2007) consideraron, tras un estudio con preadolescentes entre 7 y 12 años, que la capacidad aeróbica estaba relacionada positivamente con la flexibilidad cognitiva. Por su parte, Rigoli, Piek, Kane y Oosterlaan (2012), en un trabajo con 93 adolescentes de edades comprendidas entre los 12 y 16 años, encontraron relaciones de la coordinación motora con la memoria de trabajo y la inhibición. Del mismo modo, sobre esta última habilidad, Wu et al. (2011) encontraron resultados positivos tras realizar un estudio con 48 preadolescentes de entre 8 y 11 años, en el que relacionaban los resultados de una tarea de control cognitivo con el consumo de oxígeno máximo. Una investigación efectuada por Stroth, Kubesch, Dieterle, Ruschsow, Heim y Kiefer (2009) con adolescentes entre 13 y 14 años, evaluando la condición física mediante un test incremental en cicloergómetro, observaron que un mejor rendimiento físico estaba asociado a mejores respuestas en tests cognitivos que medían diversos aspectos relacionados con las funciones ejecutivas.

El conjunto de habilidades que constituyen las funciones ejecutivas está controlado, básicamente, por los circuitos de la corteza prefrontal (Davis et al., 2011), aunque requieren la participación de otras estructuras implicadas en el control ejecutivo como la corteza cingulada anterior, los ganglios basales, el cerebelo o el núcleo talámico dorsomedial (Goldberg y Bougakov, 2005; Verdejo-García y Bechara, 2010). A diferencia de otras áreas cerebrales, la maduración de la corteza prefrontal sucede más lentamente. Por esta razón, ciertas experiencias pueden facilitar su desarrollo en edades más avanzadas como la adolescencia (Best, 2010; Manga y Ra- mos, 2011; O'Hare y Sowell, 2008). El impacto de la práctica física sobre el rendimiento cognitivo, y en concreto sobre las funciones ejecutivas, puede proceder de las demandas cognitivas inherentes al ejercicio, los cambios fisiológicos producidos en el cerebro y las implicaciones cognitivas existentes cuando se realiza una tarea motora compleja, por lo que el tipo de ejercicio físico es una variable que hay que especificar (Best, 2010; Castelli, Hillman, Buck y Erwin, 2007; Tomporowski et al., 2008).

Los juegos colectivos pueden ser una buena herramienta para la estimulación de las funciones ejecutivas en niños y adolescentes, pues además del impacto fisiológico del propio ejercicio, requieren una continuada implicación cognitiva. De hecho, se considera que existe una mayor activación de nuestro cerebro cuando los juegos y ejercicios físicos contienen numerosas situaciones a resolver, lo que se ajusta a este tipo de tareas (Best, 2010; Ratey, 2008). En concreto, los deportes colectivos tienen implícitas tareas como colaborar con un compañero, anticiparse a las acciones de los oponentes, elaborar estrategias para alcanzar el éxito en una jugada o ser capaz de inhibir estímulos accesorios y centrarse en los importantes. En esta línea, en una investigación realizada por Cortis et al. (2011), con jugadores italianos de baloncesto categoría cadete, se puso de manifiesto la importancia de generar actividades que haya que resolver rápidamente para aumentar el control sobre la atención y otras funciones cognitivas.

En el ámbito del deporte colectivo, los juegos reducidos (small sided games) son ejercicios que actualmente se utilizan frecuentemente, como en es el caso del fútbol, como método alternativo al trabajo tradicional para mejorar aspectos fisiológicos, como el rendimiento aeróbico, así como técnicos y tácticos. Para ello, diversas variables pueden ser modificadas como las dimensiones del terreno de juego, el número de jugadores, reglas de juego u objetivos del mismo (Escolano-Pérez, Herrero-Nivela y Echeverría-Expósito, 2014; Frencken, Van DerPlaats, Visscher y Lemmink, 2013; HillHass, Coutts, Dawson y Rowsell, 2010; Hill-Hass, Rowsell, Dawson y Coutts, 2009; Sampaio, Abrantes y Leite, 2009). Como ejemplo, la intensidad del ejercicio y la percepción subjetiva del esfuerzo es inversamente proporcional al número de jugadores (Casamichana, Castellano, BlancoVillaseñor y Usabiaga, 2012; Hill-Hass, Dawson, Impellizzeri y Coutts, 2011). Además, se puede conseguir aumentar la participación, incrementando el número de intervenciones y decisiones a tomar, al disminuir el terreno de juego, el número de jugadores o el tiempo de juego (Casamichana y Castellano, 2009; Duarte, Batalha, Folgado y Sampaio, 2009; Jones y Drust, 2007).

A partir del modelo propuesto por Diamond (2006) y tras analizar la literatura existente, en la que se aprecia la necesidad de aumentar las evidencias científicas en el periodo adolescente, el presente estudio analiza los efectos de un programa de ocho semanas de actividad física sobre la memoria de trabajo, la inhibición y la flexibilidad cognitiva. 


\section{Método}

\section{Participantes}

Participaron en la investigación 54 adolescentes del municipio de Priego de Córdoba (Córdoba, España), siendo el $25.93 \%$ chicos $\left(\mathrm{n}_{1}=14\right)$ y el $74.07 \%$ chicas $\left(\mathrm{n}_{2}=40\right)$, con edades comprendidas entre los 15 y 16 años $(M \pm D T$ : edad $=15.35 \pm .48$ años; altura $=163.61 \pm 6.98 \mathrm{~cm}$; peso $=$ $61.27 \pm 10.72 \mathrm{~kg} ; \mathrm{IMC}=22.91 \pm 3.93 \mathrm{~kg} \cdot \mathrm{m}^{-2}$; masa grasa $=$ $24.42 \pm 9.95 \%$; masa magra: $45.72 \pm 7.09 \%$; FCmax $=$ $\left.198.47 \pm 6.95 \mathrm{ppm} ; \mathrm{VO} 2 \mathrm{max}=40.69 \pm 4.67 \mathrm{ml} \cdot \mathrm{kg}^{-1} \cdot \mathrm{min}^{-1}\right)$. La muestra se seleccionó de un centro escolar de dicha localidad, donde se realizó la intervención y las mediciones pertinentes. Los criterios de exclusión fueron repetir curso, realizar actividad física de forma regular (entrevista personal) y la existencia de patologías de algún tipo, lo que fue consultado al departamento de orientación de dicho centro.

\section{Instrumentos}

a) Tests Dígitos (D) y Letras y Números (LN) de la Escala de Inteligencia de Wechsler para Niños (WISC-IV; Wechsler, 2003, 2005). Se emplearon estos instrumentos para analizar la memoria de trabajo. Estas pruebas muestran un índice de memoria de trabajo (IMT), que consiste en la capacidad para mantener información de un modo activo y poder utilizarla para dar una respuesta adecuada (Swanson, 1996). La prueba Dígitos consiste en memorizar y repetir una secuencia de números, cada vez más amplia, después de que el evaluador haya leído en voz alta dicho conjunto con una cadencia de un segundo entre número y número. Un primer grupo de secuencias hay que repetirlas en el orden establecido, y un segundo conjunto hay que realizarlo en orden inverso. La prueba Letras y Números consiste en un ejercicio en el que se ofrece una secuencia desordenada de letras y números, con el mismo lapso de tiempo, y la persona que lo ejecuta debe ordenar los números en sentido ascendente y las letras en orden alfabético.

b) Trail Making Test, formas A y B (Reitan, 1958; Reitan, 1992; Reitan y Wolfson, 1985; Tombaugh, 2004). Se utilizó para analizar la flexibilidad cognitiva. La forma A está compuesta por una prueba en la que se presentan 25 números distribuidos en una hoja y los participantes tienen que conectarlos con una línea en orden ascendente, pasando de manera correlativa por todos los números. En la forma B, hay que alternar números y letras uniendo de forma ascendente y ordenada 13 números, así como las letras desde la $\mathrm{A}$ hasta la L. Antes de realizar cada una de las pruebas se permite efectuar un ensayo sobre un ejemplo diferente al ejercicio evaluado. Se ha contabilizado el tiempo, indicando al participante que debía volver al último ítem correcto cuando se equivocase. El Trail Making Test se considera apropiado para evaluar las funciones ejecutivas, siendo la forma B un buen indicador de flexibilidad cognitiva. De hecho, debe modificar una tarea similar a la que había practicado en la parte A para alternar los números con las letras. La persona que presta menos atención y es menos flexible suele cometer más errores durante su ejecución (Soprano, 2003).

c) Test Stroop (Stroop, 1935; Golden, 1994). Se empleó para evaluar el control inhibitorio. Esta prueba trata de evaluar la capacidad para seleccionar información, inhibiendo respuestas automáticas y formulando la respuesta correcta (Soprano, 2003). Consta de 3 partes, palabras, colores y palabras/colores. En la primera, se presenta una lista de 100 palabras, en la que el ejecutante debe decir el mayor número de ellas en 45 segundos, empezando de nuevo si no se ha consumido el tiempo aún cuando llega a la última. De igual forma, en la segunda lámina se presenta el texto XXXX escrito en un color determinado, en otra lista de 100 elementos. Hay que resolverlo indicando, en este caso, el color en que está escrito y siguiendo el procedimiento anterior. En la tercera hoja, se presentan 100 palabras que indican un color pero escritas en otro diferente, debiéndose decir el color en el que están impresas. En esta tercera lámina, el efecto interferencia que genera el color que indica la palabra con el que está escrito debe ser solventado por el que ejecuta la prueba para resolverla con éxito.

d) Evaluación de la condición física para clasificar y aleatorizar la muestra. Se ha utilizado una prueba de la batería EUROFIT (1993), el test de Course Navette, para calcular de forma indirecta el consumo máximo de oxígeno (VO2max). El test de Course Navette consiste en un test incremental de ida y vuelta, sobre una distancia de 20 metros, con un aumento de la velocidad de $0.5 \mathrm{~km}$ cada minuto, con una velocidad inicial de $8.5 \mathrm{~km} / \mathrm{h}$. Para el cálculo concreto del consumo de oxígeno se aplicó la fórmula $V O 2 \max =$ $31.025+3.238 \mathrm{~V}-3.248 \mathrm{E}+0.1536 \mathrm{VE}$ (siendo $V$ la velocidad alcanzada en la última etapa completada y $E$ la edad del participante). Además, se han obtenido valores de la frecuencia cardíaca máxima (FCmax) y la FC de trabajo para el control de la actividad. Para ello, se han utilizado pulsómetros Po$\operatorname{lar}{ }^{\circledR}$ modelo S610, que registra frecuencia cardiaca cada 5 segundos. También se ha empleado un bioimpedanciómetro (Tanita ${ }^{\circledR}$ Body Composition Monitor modelo BF-350) para medir el porcentaje de masa grasa, magra e índice de masa corporal, introduciendo previamente la altura. El modelo utilizado, que posee electrodos en 4 puntos de contacto para la planta del pie, es un aparato funcional que mediante señal de baja frecuencia permite obtener medidas instantáneas de porcentaje de agua y grasa corporal, peso óseo, masa muscular, índice metabólico basal o masa muscular, entre otros.

\section{Procedimiento}

Inicialmente, se informó mediante carta al centro escolar y a los padres sobre el estudio que se pretendía realizar. Se obtuvo consentimiento informado de los padres y el permiso de la dirección del centro. Además, durante el proceso de la investigación, se respetaron los principios de la declaración de Helsinki. Se realizaron dos evaluaciones a todos los participantes, antes de comenzar el programa y al finalizarlo, 
empleándose aproximadamente 30 minutos para cada uno de ellos en las pruebas neuropsicológicas y las medidas de composición corporal. Además, en grupos de 12-14 se realizó el test de Course Navette, siendo necesario 20 minutos para cada uno. Se contó con la participación de psicólogos especializados y varios colaboradores, que desconocían la pertenencia de los participantes al grupo control o experimental. Al analizar la composición corporal, y para que los resultados obtenidos con la Tanita ${ }^{\circledR}$ BF-350 tuvieran un mínimo margen de error, se tuvieron en cuenta una serie de pautas y recomendaciones, como las propuestas por George, Fisher, y Vehrs (2007) para la estimación de la composición corporal. El protocolo a seguir fue: no realizar ejercicios agotadores el día anterior, no alterar significativamente la alimentación el día previo a la prueba, vestir ropa cómoda, controlar la toma de medicamentos que puedan alterar los niveles de agua corporal y evitar retener líquidos.

Tras la primera toma de datos, se distribuyó a los participantes al azar en los grupos control y experimental, teniendo en cuenta las variables género, composición corporal y consumo de oxígeno máximo. Se intervino durante 8 semanas sobre el grupo experimental, aumentando la práctica física semanal 90 minutos, divididos en una sesión de 60 y otra de 30 minutos, pasando de 120 (clases de educación física ordinarias) a 210 minutos semanales. Estas sesiones se implementaron en días diferentes a las clases de Educación Física, lo que hizo que los integrantes del grupo experimental practicaran actividad física 4 días a la semana en horario escolar. Durante el programa, ambos grupos siguieron las sesiones programadas en clases de educación física, que estuvieron divididas en un primer bloque de deportes colectivos (enseñanza del baloncesto) y un segundo de expresión corporal (aerobic y bailes modernos).

Diversos colaboradores estuvieron presentes durante la intervención para ayudar al control del juego y asegurar el máximo tiempo útil posible. En la sesión de 60 minutos, se efectuaron 6 juegos reducidos 3x3 (2 de fútbol sin portero, 2 de baloncesto y 2 de balonmano sin portero) con una duración de 6 minutos cada uno y un descanso de 1 minuto. En la sesión de 30 minutos, se efectuaron 3 juegos en espacios reducidos $3 \times 3$ (1 de fútbol sin portero, 1 de baloncesto y 1 de balonmano sin portero) con una duración de 6 minutos cada uno y un descanso de 1 minuto. Se efectuó un calentamiento de 7 minutos en cada una de las sesiones, que consistió en ejercicios de activación, movilidad articular y en un juego $3 \times 3$ en el que había que mantener la posesión de un balón de gomaespuma utilizando las manos. El área de juego total fue, para cada ejercicio, de $240 \mathrm{~m}^{2}(20 \times 12 \mathrm{~m})$, siendo el área relativa por jugador de $40 \mathrm{~m}^{2}$. La frecuencia cardiaca media con la que se participó fue de $175.96 \pm 10.26 \mathrm{ppm}$ $(81.03 \pm 7.82 \% \mathrm{FCR})$ y una percepción media de esfuerzo (Borg) de $13.36 \pm 1.39$.

\section{Análisis de los datos}

Para valorar el propósito principal del estudio, se realizó un ANOVA factorial mixto para cada medida cognitiva (memoria de trabajo, inhibición y flexibilidad cognitiva) definidas como variables dependientes. Como variables independientes se definieron el grupo con dos niveles (control y experimental) manipulados intersujeto y la variable pre-post con dos niveles y manipulados intrasujeto. Se estudiaron los efectos principales y la interacción entre variables. La significación de cada efecto se analizó mediante comparaciones de Bonferroni. También se realizaron pruebas t-student, tras analizar la normalidad con la prueba de Kolmogorov-Smirnov, para analizar la homogeneidad de los grupos en diversas variables previas al programa de actividad física. De igual forma se estudió la homogeneidad de los grupos en función del género, aplicando pruebas t-student en chicas y $U$ Mann-Whitney en chicos (por ser un grupo pequeño). El programa estadístico utilizado fue el SPSS en su versión 20.

\section{Resultados}

\section{Descriptivos previos y homogeneidad de los grupos}

En la Tabla 1 se pueden observar los valores de condición física de los grupos control y experimental obtenidos

Tabla 1. Medidas previas al programa de actividad física, prueba de normalidad (Kolmogorov-Smirnov) y comparación de medias $(t-s t u d e n t)$.

\begin{tabular}{|c|c|c|c|c|c|c|c|}
\hline & & $M$ & $D T$ & As & $K$ & $Z$ & $T$ \\
\hline \multirow[t]{2}{*}{ Masa grasa } & Control & 25.52 & 10.27 & -.27 & -1.17 & $.56^{\mathrm{a}}$ & \multirow{2}{*}{$1.12^{\mathrm{a}}$} \\
\hline & Experimental & 22.65 & 8.34 & -.10 & -.89 & $.52^{\mathrm{a}}$ & \\
\hline \multirow[t]{2}{*}{ Masa magra } & Control & 45.58 & 6.5 & 1.06 & 1.35 & $.89^{a}$ & \multirow{2}{*}{$-.14^{a}$} \\
\hline & Experimental & 45.86 & 7.73 & .81 & -.04 & $.91^{a}$ & \\
\hline \multirow[t]{2}{*}{ IMC } & Control & 23.54 & 4.24 & .65 & -.02 & $.72^{\mathrm{a}}$ & \multirow{2}{*}{$1.18^{\mathrm{a}}$} \\
\hline & Experimental & 22.28 & 3.56 & .59 & -.25 & $.60^{\mathrm{a}}$ & \\
\hline \multirow[t]{2}{*}{$\mathrm{VO} 2 \max$} & Control & 40.46 & 4.82 & .02 & -1.38 & $.85^{a}$ & \multirow{2}{*}{$-.36^{\mathrm{a}}$} \\
\hline & Experimental & 40.92 & 4.59 & .13 & -1.12 & $.80^{\mathrm{a}}$ & \\
\hline \multirow[t]{2}{*}{ FCmax } & Control & 197.75 & 1.42 & .58 & .25 & $.59^{\mathrm{a}}$ & \multirow{2}{*}{$.29 \mathrm{a}$} \\
\hline & Experimental & 197.21 & 1.94 & -.52 & 1.36 & $.64^{\mathrm{a}}$ & \\
\hline
\end{tabular}

$\mathrm{a} p>.05$.

$I M C=$ Indice de masa corporal; VO2max= Consumo de oxígeno máximo; FCmax = Frecuencia cardíaca máxima. As= Asimetría; $K=$ Kurtosis.

tras la distribución de los participantes. Se realizó análisis de normalidad mediante la prueba de Kolmogorov-Smirnov y análi-

sis de la asimetría y curtosis. La prueba de comparación de medias empleada (t-student) indicó la homogeneidad existente 
entre los grupos al no hallarse diferencias significativas. En la Tabla 2 se pueden observar los valores de condición física para ambos grupos en función del género. Las pruebas de comparación de medias empleadas ( $t$-student, para chicas, y $U$ Mann-Whitney, para chicos) indicaron que los grupos eran homogéneos.

Tabla 2. Medidas previas al programa de actividad física en función del género.

\begin{tabular}{|c|c|c|c|c|c|c|c|}
\hline & & \multicolumn{3}{|c|}{ Chicos } & \multicolumn{3}{|c|}{ Chicas } \\
\hline & & $M$ & $D T$ & $Z$ & $M$ & $D T$ & $T$ \\
\hline \multirow[t]{2}{*}{ Masa grasa } & Control & 13.20 & 5.18 & \multirow[b]{2}{*}{$-.06^{\mathrm{a}}$} & 29.83 & 7.76 & \multirow{2}{*}{$1.86^{\mathrm{a}}$} \\
\hline & Experimental & 14.24 & 7.40 & & 25.59 & 6.54 & \\
\hline \multirow[t]{2}{*}{ Masa magra } & Control & 53.34 & 6.87 & \multirow{2}{*}{$-.71^{a}$} & 42.86 & 3.67 & \multirow{2}{*}{$.39^{\mathrm{a}}$} \\
\hline & Experimental & 55.82 & 6.41 & & 42.36 & 4.40 & \\
\hline \multirow[t]{2}{*}{ IMC } & Control & 21.01 & 2.64 & \multirow{2}{*}{$-.70^{a}$} & 24.43 & 4.37 & \multirow{2}{*}{$1.62^{\mathrm{a}}$} \\
\hline & Experimental & 21.98 & 3.76 & & 22.38 & 3.57 & \\
\hline \multirow[t]{2}{*}{$\mathrm{VO} 2 \max$} & Control & 45.63 & 2.33 & \multirow{2}{*}{$-.61^{\mathrm{a}}$} & 38.65 & 4.24 & \multirow{2}{*}{$-.54^{a}$} \\
\hline & Experimental & 45.44 & 3.91 & & 39.34 & 3.73 & \\
\hline \multirow[t]{2}{*}{ FCmax } & Control & 202.57 & 6.53 & \multirow{2}{*}{$-1.29^{a}$} & 195.85 & 5.63 & \multirow[t]{2}{*}{$-.23^{a}$} \\
\hline & Experimental & 198.86 & 6.91 & & 196.35 & 7.21 & \\
\hline
\end{tabular}

$p>.05$

$I M C=$ Indice de masa corporal; VO2max= Consumo de oxígeno máximo; FCmax= Frecuencia cardiaca máxima .

\section{Evaluación cognitiva}

En la Tabla 3 se muestran los análisis descriptivos para cada medida y grupo, así como los resultados de la prueba de Levene para analizar la homogeneidad de las varianzas. Como se puede apreciar, no hubo problemas de homogeneidad de varianza en ninguna prueba tanto antes como después de la intervención.

Tabla 3. Estadísticos descriptivos de las pruebas de evaluación cognitiva.

\begin{tabular}{|c|c|c|c|c|c|c|}
\hline & & \multicolumn{2}{|c|}{ Control } & \multicolumn{2}{|c|}{ Experimental } & \multirow[b]{2}{*}{ Levene } \\
\hline & & $M$ & $D T$ & $M$ & $D T$ & \\
\hline \multirow[t]{2}{*}{ D } & Pre & 14.81 & 2.62 & 15.82 & 1.92 & $1.88^{\mathrm{a}}$ \\
\hline & Post & 16.07 & 2.22 & 18.15 & 2.07 & $.06^{\mathrm{a}}$ \\
\hline \multirow[t]{2}{*}{ LN } & Pre & 16.44 & 2.41 & 17.52 & 2.28 & $.01^{\mathrm{a}}$ \\
\hline & Post & 17.37 & 2.62 & 19.44 & 1.83 & $2.76^{\mathrm{a}}$ \\
\hline \multirow[t]{2}{*}{ IMT } & Pre & 14.33 & 5.01 & 17.01 & 4.20 & $.04^{a}$ \\
\hline & Post & 16.78 & 4.86 & 21.22 & 3.51 & $3.18^{a}$ \\
\hline \multirow[t]{2}{*}{ TMT-A } & Pre & 28.25 & 10.32 & 26.39 & 7.37 & $.99 a$ \\
\hline & Post & 25.93 & 6.62 & 21.58 & 6.18 & $.67 \mathrm{a}$ \\
\hline \multirow[t]{2}{*}{ TMT-B } & Pre & 64.09 & 17.23 & 65.18 & 16.53 & $.35^{\mathrm{a}}$ \\
\hline & Post & 58.52 & 16.51 & 51.01 & 12.23 & $2.56^{\mathrm{a}}$ \\
\hline \multirow[t]{2}{*}{ TS-P } & Pre & 104.48 & 10.02 & 108.04 & 11.49 & $.28^{a}$ \\
\hline & Post & 107.93 & 9.96 & 115.01 & 12.77 & $.51^{\mathrm{a}}$ \\
\hline \multirow[t]{2}{*}{ TS-C } & Pre & 71.11 & 9.17 & 75.48 & 10.41 & $.12^{a}$ \\
\hline & Post & 73.59 & 9.65 & 80.96 & 12.39 & $1.04^{\mathrm{a}}$ \\
\hline \multirow[t]{2}{*}{ TS-PC } & Pre & 43.04 & 9.98 & 46.07 & 8.99 & $.33^{a}$ \\
\hline & Post & 50.44 & 10.99 & 51.74 & 9.53 & $.01^{\mathrm{a}}$ \\
\hline
\end{tabular}

${ }^{\mathrm{a} p}>.05$

$D=$ Test de Digitos; $L N=$ Test de Letras y Números; IMT= Índice de Memoria de Trabajo; TMT-A= Trail Making Test forma A; TMT-B= Trail Making Test forma B; TS$P=$ Test Stroop Palabras; TS-C= Test Stroop Colores; TS-PC= Test Stroop Palabras-Colores.

En la Tabla 4 se muestran los resultados de los ANOVAs factoriales mixtos realizados, para el test de Dígitos, Test de Letras y Números, Índice de Memoria de Trabajo, Trail Making Test (A y B) y Test Stroop (palabras, colores y palabras/colores). Como se aprecia, existieron diferencias significativas en los efectos principales de la variable pre-post para todas las medidas evaluadas, y de la variable grupo en el test de Digitos, test de Letras y Números, Índice de Memoria de Tra- bajo, Test Stroop palabras y Test Stroop colores. Por otro lado, en los efectos de interacción, hubo diferencias significativas en las medidas de los tests Dígitos $\left(F_{[1,52]}=6.47 ; p<.05 ; \eta^{2}=.11\right.$; $1-\beta=.70)$, Letras y Números $\left(F_{[1,52]}=4.76 ; \mathrm{p}<.05 ; \eta^{2}=.09 ; 1\right.$ $\beta=.57)$, en el Índice de Memoria de Trabajo $\left(F_{[1,52]}=4.52 ; p<.05\right.$; $\left.\eta^{2}=.08 ; 1-\beta=.55\right)$ y en el Trail Making Test forma $B\left(F_{[1,52]}=\right.$ $\left.4.12 ; p<.05 ; \eta^{2}=.07 ; 1-\beta=.51\right)$. 
Tabla 4. Resultados de los ANOVAs factoriales mixtos para cada prueba de evaluación cognitiva. Se muestran los valores de la $F$, el valor $p$, el tamaño del efecto $\left(\eta^{2}\right)$ y la potencia del contraste $(1-\beta)$.

\begin{tabular}{|c|c|c|c|c|}
\hline & & Pre-post & Grupo & Interacción \\
\hline \multirow[t]{3}{*}{ Test Dígitos } & $F$ & $72.38^{* * *}$ & $7.36^{* *}$ & $6.47^{*}$ \\
\hline & $\eta^{2}$ & .58 & .12 & .11 \\
\hline & $1-\beta$ & 1.00 & .76 & .70 \\
\hline \multirow[t]{3}{*}{ Test Letras y Números } & $F$ & $35.38^{* * *}$ & $7.13^{* *}$ & $4.76^{*}$ \\
\hline & $\eta^{2}$ & .41 & .12 & .09 \\
\hline & $1-\beta$ & 1.00 & .75 & .57 \\
\hline \multirow[t]{3}{*}{ Índice de Memoria de Trabajo } & $F$ & $63.50^{* * *}$ & $9.87 * *$ & $4.52 *$ \\
\hline & $\eta^{2}$ & .55 & .16 & .08 \\
\hline & $1-\beta$ & 1.00 & .87 & .55 \\
\hline \multirow[t]{3}{*}{ Trail Making Test A } & $F$ & $10.15^{* *}$ & 2.96 & 1.23 \\
\hline & $\eta^{2}$ & .16 & .05 & .02 \\
\hline & $1-\beta$ & .88 & .39 & .19 \\
\hline \multirow[t]{3}{*}{ Trail Making Test B } & $\dot{F}$ & $21.73 * * *$ & .75 & $4.12^{*}$ \\
\hline & $\eta^{2}$ & .29 & .02 & .07 \\
\hline & $1-\beta$ & .99 & .14 & .51 \\
\hline \multirow[t]{3}{*}{ Test Stroop Palabras } & $\dot{F}$ & $24.87 * * *$ & 3.50 & 2.84 \\
\hline & $\eta^{2}$ & .32 & .06 & .05 \\
\hline & $1-\beta$ & .99 & .45 & .38 \\
\hline \multirow[t]{3}{*}{ Test Stroop Colores } & $F$ & $26.07 * * *$ & $4.58^{*}$ & $3.70^{\mathrm{a}}$ \\
\hline & $\eta^{2}$ & .33 & .08 & .07 \\
\hline & $1-\beta$ & .99 & .56 & .47 \\
\hline \multirow[t]{3}{*}{ Test Stroop Palabras / Colores } & $F$ & $34.03 * * *$ & .78 & .60 \\
\hline & $\eta^{2}$ & .40 & .02 & .01 \\
\hline & $1-\beta$ & 1.00 & .14 & .12 \\
\hline
\end{tabular}

$* p<.05 ; * * p<.01 ; * * * p<.001$.

${ }^{\mathrm{a}} p=.060$.

Como se puede observar en la Tabla 5, el grupo experimental mejoró las puntuaciones en todas las pruebas tras la intervención. De igual forma, el grupo control también mejoró los resultados, aunque en el Trail Making Test las diferencias no fueron significativas. Atendiendo a los valores pretest, no hubo diferencias significativas entre los grupos control y experimental salvo en el valor Índice de Memoria de Trabajo. En el postest, si hubo diferencias significativas en todos los casos entre los grupos, menos en la puntuación del Test Stroop palabras/colores. En la Figura 1 se muestran los resultados pre y post de ambos grupos en aquellas medidas cuya interacción fue significativa.

Tabla 5. Comparaciones entregrupos e intragrupos para cada prueba de evaluación cognitiva.

\begin{tabular}{|c|c|c|c|c|}
\hline & \multicolumn{2}{|c|}{ Grupo } & \multicolumn{2}{|c|}{ Factor } \\
\hline & $\begin{array}{c}\text { Control } \\
\text { Pre vs. Post }\end{array}$ & $\begin{array}{l}\text { Experimental } \\
\text { Pre vs. Post }\end{array}$ & $\begin{array}{l}\text { Pretest } \\
\text { C vs. E }\end{array}$ & $\begin{array}{l}\text { Postest } \\
\text { C vs. E }\end{array}$ \\
\hline $\mathrm{D}$ & $-1.26 * * *$ & $-2.33 * * *$ & -1.01 & $-2.08 * * *$ \\
\hline $\mathrm{LN}$ & $-.93 *$ & $-2.00 * * *$ & -1.08 & $-2.07 * * *$ \\
\hline IMT & $-2.45^{* * *}$ & $-4.21 * * *$ & $-2.67^{*}$ & $-4.44 * * *$ \\
\hline TMT - A & 2.32 & $4.80 * *$ & 1.86 & $4.35^{*}$ \\
\hline TMT - B & $5.57^{a}$ & $14.17 * * *$ & 1.08 & $7.52^{*}$ \\
\hline TS-P & $-3.44 *$ & $-6.96 * * *$ & -3.56 & $-7.07^{*}$ \\
\hline TS-C & $-2.48^{*}$ & $-5.48 * * *$ & -4.37 & $-7.37 *$ \\
\hline TS-PC & $-7.41 * * *$ & $-5.67 * * *$ & -3.04 & -1.29 \\
\hline
\end{tabular}

$* p<.05 ; * * p<.01 ; * * * p<.001$

$\mathrm{a} p=.068$

$D=$ Test de Digitos; $L N=$ Test de Letras y Números; IMT= Índice de Memoria de Trabajo; TMT-A= Trail Making Test forma A; TMT-B= Trail Making Test forma B; TS $P=$ Test Stroop Palabras; TS $-C=$ Test Stroop Colores; TS-PC $=$ Test Stroop Palabras-Colores. 


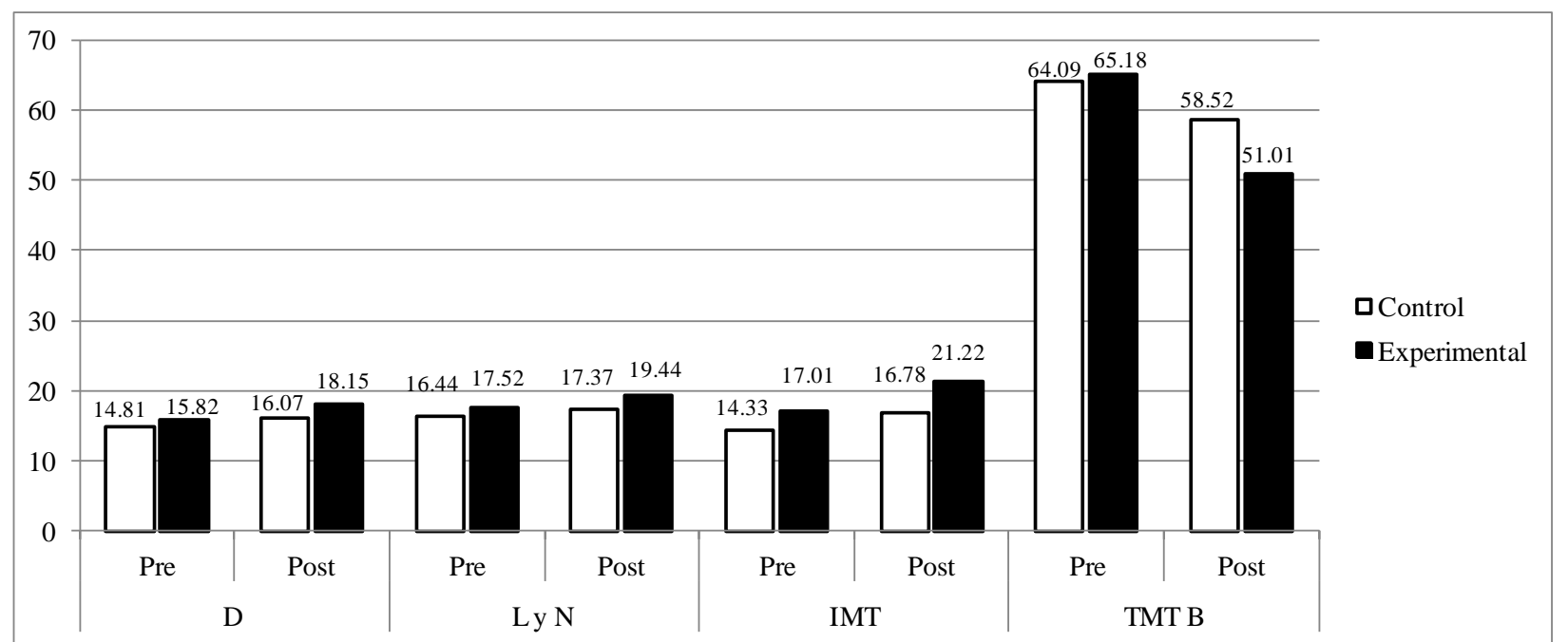

Figura 1. Gráfico de las puntuaciones obtenidas en el Test de Dígitos, Test de Letras y Números, Índice de memoria de trabajo y Trail Making Test, en función de las variables grupo y medidas pre-post.

\section{Discusión}

Este trabajo pretendía poner de manifiesto los efectos de un programa de ejercicio físico sobre diversas variables vinculadas a las funciones ejecutivas, como el control del impulso en la respuesta, memoria de trabajo y flexibilidad cognitiva. Los resultados obtenidos han puesto de manifiesto efectos positivos de la intervención en las medidas memoria de trabajo y flexibilidad cognitiva, lo que satisface el objetivo de la investigación. Aunque el efecto del programa no se aprecia en todas las variables y el tamaño del mismo no es muy elevado, la duración del periodo de intervención, que no fue excesivamente extenso, y la edad de los participantes, adolescencia media, permiten considerar interesantes los resultados hallados en este trabajo.

Los resultados obtenidos se encuentran en la línea de otros estudios que han analizado los efectos de la práctica de actividad física sobre variables de índole cognitiva en niños y adolescentes (Chaddock, Pontifex, Hillman y Kramer, 2011; Hillman et al., 2008; Hillman, Kamijo y Scudder, 2011), y en concreto aquellos que han estudiado aspectos específicos de las funciones ejecutivas (Best, 2010; Davis et al., 2011; Tomporowski et al., 2008; Tomporowski et al., 2011). Hay que destacar que la mayoría de las investigaciones realizadas al respecto se han efectuado con muestras de edades inferiores a la de este trabajo, siendo difícil encontrar estudios que analicen este fenómeno con grupos situados en la adolescencia media y avanzada. En este sentido, existen evidencias que ponen de manifiesto la evolución que experimentan las funciones ejecutivas a lo largo de toda la adolescencia o, incluso, la juventud temprana. Éstas se fundamentan en el desarrollo que continúa teniendo el cerebro humano en estas edades, sobre todo en áreas como la corteza prefrontal (Blakemore y Choudhury, 2006; O'Hare y Sowell, 2008; Powell y Voeller, 2004; Tomporowski et al., 2011), lo que hace viable el estu- dio sobre el impacto que puede tener la actividad física en edades adolescentes más avanzadas.

En este tipo de trabajos es importante seleccionar con precisión las variables que se van a evaluar dado que el proceso madurativo de los niños y adolescentes determina las capacidades sobre las que se podrá incidir más. Como sugieren Tomporowski et al. (2008) hay aspectos que se pueden seguir estimulando en edad escolar media, como la memoria de trabajo, pero otras como el control inhibitorio será más complicado en edades avanzadas (Best, Miller y Jones, 2009). Este tipo de factores han podido influir en los resultados encontrados, dado que los cambios en las tareas en las que estaba implicada la memoria de trabajo han resultado significativos, pero en aquellas que valoraban el control de la impulsividad han sido menos favorables. Determinar con precisión este tipo de aspectos debe ser una línea prospectiva de investigación. De hecho, concretar las relaciones causales específicas entre la actividad física y las habilidades cognitivas estudiadas, así como la edad apropiada de aplicación, es necesario para poder utilizar la práctica física como una herramienta con verdadero valor en este tipo de procedimientos (Kramer y Erickson, 2007).

Por otro lado, tal y como señalan autores como Fedewa y Ahn (2011) o Tomporowski et al. (2008), dentro del conjunto de programas de actividad física que se han llevado a cabo para mejorar las habilidades cognitivas, aquellos que se centran en trabajar aspectos vinculados al rendimiento aeróbico son los que han dado mejores resultados. La intervención llevada a cabo en este trabajo se ha realizado con juegos reducidos $3 \times 3$, estando ampliamente estudiado que este tipo de actividades contribuyen a trabajar y desarrollar dicha capacidad (Hill-Hass et al., 2011). Aunque no se han realizado pruebas físicas para observar cambios en esta capacidad, lo que se convierte en una limitación del estudio y futura línea de trabajo, sí se han obtenido datos sobre la frecuencia cardiaca media durante la realización de los ejercicios, que indi- 
can que se estuvo trabajando a una intensidad adecuada para dicho objetivo.

Además, Diamond y Lee (2011) sugieren que la actividad física desarrollada en el contexto deportivo puede tener un mayor impacto sobre el funcionamiento cerebral, dada la gran implicación cognitiva que tienen estas situaciones, derivadas de la alta intensidad del juego, e incluso por las implicaciones socioemocionales que conllevan. En el marco de la actividad deportiva, el trabajo con juegos en espacios reducidos $3 \times 3$ genera un mayor número de intervenciones que otras versiones con un mayor número de participantes, incrementando la cantidad de estímulos a los que hay que atender y los problemas que hay que resolver (Dellal, Drust y Lago-Penas, 2012; Dellal, Hill-Hass, Lago-Penas y Chamari, 2011). Katis y Kellis (2009) consideran, en concreto, que el juego $3 \times 3$ tiene ventajas sobre otros con mayor número de participantes y lo aconsejan para mejorar la condición física y las habilidades técnicas. En su conjunto, estos factores se encontrarían en la base que, según diversos investigadores, explicarían los efectos de este tipo de ejercicios sobre las habilidades cognitivas (Best, 2010).

Estos resultados, aportan nuevos datos sobre este fenómeno y sugieren que se debe continuar profundizando en aquellos aspectos de la actividad física que pueden incidir en el desarrollo cognitivo de los niños y adolescentes. De este modo, hay que hacer hincapié en la necesidad de valorar el papel de la asignatura de Educación Física en la escuela, debiéndose incrementar el tiempo dedicado a esta asignatura en los centros escolares. De hecho, los hallazgos encontrados en el área del desarrollo cognitivo indican la necesidad de que la Educación Física sea una parte importante del currículum de los alumnos (Berg, 2010). Además, esta necesidad se hace más evidente cuando los resultados de encuestas sobre la práctica física frecuente en niños y adolescentes indican niveles elevados de sedentarismo, afectando también a otros aspectos que pueden influir en el rendimiento cognitivo de los adolescentes como es la obesidad (Moral-García, Redecillas-Peiró, y Martínez-López, 2012; Ramos, Rivera,

\section{Referencias}

Banich, M.T. (2009). Executive Function: The search for an integrated account. Current Directions in Psychological Science, 18(2), 89-94.

Berg, K. (2010). Justifying physical education based on neuroscience evidence. Journal of Physical Education, Recreation and Dance, 81(3), 24-29.

Best, J.R. (2010). Effects of physical activity on children's executive function: Contributions of experimental research on aerobic exercise. Developmental Review, 30(4), 331-351.

Best, J.R., Miller, P.H. y Jones, L.L. (2009). Executive functions after age 5: Changes and correlates. Developmental Review, 29(3), 180-200.

Blakemore, S.J. y Choudhury, S. (2006). Development of the adolescent brain: implications for executive function and social cognition. Journal of Child Psychology and Psychiatry, 47(3), 296-312.

Buck, S.M., Hillman, C.H. y Castelli, D.M. (2008). The relation of aerobic fitness to stroop task performance in preadolescent children. Medicine \& Science in Sports \& Exercise, 40(1), 166-172.

Burgess, P.W., Alderman, N., Forbes, C., Costello, A., Coates, L.M., Dawson, D.R., ... Channon, S. (2006). The case for the development and use of "ecologically valid" measures of executive function in experi-
Moreno, y Jiménez-Iglesias, 2012; Verdejo-García et al., 2010).

Como ya se ha comentado, este trabajo presenta algunas limitaciones, que sin desvirtuar los hallazgos encontrados, permiten plantear nuevas acciones en futuras investigaciones. En primer lugar, se podrían aumentar los periodos de intervención y realizar mediciones intermedias, para observar los cambios en el tiempo. También sería interesante evaluar, paralelamente, los cambios físicos y fisiológicos ocurridos durante la intervención, para intentar determinar su impacto específico y, de esta manera, poder diferenciarlo del efecto que pueden tener otros factores como el entrenamiento cognitivo al que está sometido un participante en dichas actividades. Además, se podría comparar el efecto de los juegos en espacios reducidos con ejercicios en situaciones de práctica real, para observar si la mayor implicación y participación que acontece en los small sided games es un factor determinante para este tipo de variables. En cualquier caso, y a pesar de la necesidad de mejorar ciertos aspectos, el presente estudio aporta nuevos datos sobre el impacto que la actividad física tiene sobre la cognición, en concreto sobre las funciones ejecutivas.

\section{Conclusiones}

Los resultados obtenidos en esta investigación indican que el programa de actividad física aplicado ha tenido un efecto positivo sobre el Índice de Memoria de Trabajo de la Escala de Inteligencia de Wechsler para Niños (WISC-IV) y el tiempo en realizar las pruebas Trail Making Test B. Esto contribuye a consolidar la importancia de la actividad física regular sobre el desarrollo cognitivo de los adolescentes y muestra una forma de trabajo que puede resultar adecuada (small sided games) para conseguir estos efectos. Los datos hallados sugieren la importancia de aumentar la práctica física en los centros educativos, dados los beneficios que de ella se pueden derivar, y la necesidad de que los profesionales adapten sus programas con aquellas actividades que contribuyan a potenciar estos efectos.

mental and clinical neuropsychology. Journal of the International Neuropsychological Society, 12(2), 194-209.

Casamichana, D. y Castellano, J. (2009). Análisis de los diferentes espacios individuales de interacción y los efectos en las conductas motrices de los jugadores. Aplicaciones al entrenamiento en fútbol. Motricidad. European Journal of Human Movement, 15(23), 143-167.

Casamichana, D., Castellano, J., Blanco-Villaseñor, A. y Usabiaga, O. (2012). Estudio de la percepción subjetiva del esfuerzo en tareas de entrenamiento en fútbol a través de la teoría de la generalizabilidad. Revista de Psicología del Deporte, 21(1), 35-40.

Castelli, D.M., Hillman, C.H., Buck, S.M. y Erwin, H. (2007). Physical fitness and academic achievement in 3rd and 5th grade students. Journal of Sport \& Exercise Psychology, 29(2), 239-252.

Chaddock, L., Erickson, K.I., Prakash, R.S., Kim, J.S., Voss, M.W., VanPatter, M., ... Kramer, A.F. (2010). A neuroimaging investigation of the association between aerobic fitness, hippocampal volume and memory performance in preadolescent children. Brain Research, 1358, 172-83. 
Chaddock, L., Hillman, C.H., Pontifex, M.B., Jonhson, C.R., Raine, L.B. y Kramer, A.F. (2011). Childhood aerobic fitness predicts cognitive performance one year later. Journal of Sport Sciences, 30(5), 421-430.

Chaddock, L., Pontifex, M.B., Hillman, C.H. y Kramer, A.F. (2011). A Review of the Relation of Aerobic Fitness and Physical Activity to Brain Structure and Function in Children. Journal of the International Neuropsychological Society, 17(6), 1-11.

Cortis, C., Tessitore, A., Lupo, C., Pesce, C., Fossile, E., Figura, F. y Capranica, L. (2011). Inter-limb coordination and strength, jump, and sprint performances following a youth men's basketball game. Journal of Strength and Conditioning Research, 25(1), 135-142.

Davidson, M.C., Amso, D., Anderson, L.C. y Diamond, A. (2006). Development of cognitive control and executive functions from 4 to 13 years: Evidence from manipulations of memory, inhibition, and task switching. Neuropsychologia, 44(11), 2037-2078.

Davis, C.L., Tomporowski, P.D., McDowell, J E., Austin, B.P., Miller, P.H., Yanasak, N.E., ... Naglieri, J.A. (2011). Exercise improves executive function and achievement and alters brain activation in overweight children: a randomized, controlled trial. Health psychology: official journal of the Division of Health Psychology, American Psychological Association, 30(1), 91-98.

Dellal, A., Drust, B. y Lago-Penas, C. (2012). Variation of activity demands in small-sided soccer games. International Journal of Sport Medicine, 33(5), 370-375.

Dellal, A., Hill-Haas, S., Lago-Penas, C. y Chamari, K. (2011). Small-sided games in soccer: Amateur vs. professional players' physiological responses, physical, and technical activities. Journal of Strength and Conditioning Research, 25(9), 2371-2381.

Diamond, A. (2006). The early development of executive functions. En E. Bialystok y F. I. Craik (Eds.), Lifespan cognition: Mechanisms of change (pp. 70-95). Oxford: Oxford University Press.

Diamond, A. y Lee, K. (2011). Interventions shown to aid executive function development in Children 4-12 years old. Science, 133(6045), 959964.

Duarte, R., Batalha, N., Folgado, H. y Sampaio, J. (2009). Effects of exercise duration and number of players in heart rate responses and technical skills during futsal small-sided games. The Open Sports Sciences Journal, 3(2), 13-15.

Escolano-Pérez, E., Herrero-Nivela, M.L. y Echeverría-Expósito, R. (2014). La resolución de problemas como estrategia de enseñanza para el aprendizaje de la táctica ofensiva en la categoría pre-benjamín de fútbol 8. Revista de Psicología del Deporte, 23(1), 209-217.

Eurofit (1993). Eurofit Tests of Physical Fitness (2 ${ }^{\mathrm{a}}$ ed.). Strasbourg: Committee of Experts on Sports Research.

Fedewa, A.L. y Ahn, S. (2011). The effects of physical activity and physical fitness on children's achievement and cognitive outcomes: a metaanalysis. Research Quarterly For Exercise and Sport, 82(3), 521-535.

Frencken, W., Van DerPlaats J., Visscher, C. y Lemmink, K. (2013). Size matters: pitch dimensions constrain interactive team behaviour in soccer. Journal of Systems Science and Complexity, 26(1), 85-93.

George, J.D., Fisher, A.G. y Vehrs, P.R. (2007). Tests y pruebas fisicas $\left(4^{a} \mathrm{ed}\right.$.). Barcelona: Paidotribo.

Goldberg, E. y Bougakov, D. (2005). Valoración neuropsicológica de la disfunción del lóbulo frontal. Psychiatric Clinics of North America, 28(3), $567-$ 580 .

Golden, C.J. (1994). Stroop: Test de colores y palabras. Madrid: TEA Ediciones.

Gómez-Cabello, A., Ara, I., González-Agüero, A., Casajús, J. A. y VicenteRodríguez, G. (2012). Effects of Training on Bone Mass in Older Adults. Sports Medicine, 42(4), 301-325.

Hill-Hass, S.V., Coutts, A.J., Dawson, B.T. y Rowsell, G.J. (2010). Timemotion characteristics of physiological response of small-sided games in elite youth players: The influence of player number and rule changes. Journal of Strength and Conditioning Research, 24(8), 2149-2156.

Hill-Hass, S.V., Dawson, B.T., Impellizzeri, F.M. y Coutts, A.J. (2011). Physiology of small-sided games training in football. Sports Medicine, 41(3), 199-220.

Hill-Hass, S.V., Rowsell, G.J., Dawson, B.T. y Coutts, A.J. (2009). Acute physiological responses and time-motion characteristics of two smallsided training regimes in youth soccer players. Journal of Strength and Conditioning Research, 23(1), 111-115.
Hillman, C.H., Erickson, K.I. y Kramer, A.F. (2008). Be smart, exercise your heart: exercise effects on brain and cognition. Nature Reviews Neuroscience, 9(1), 58-65.

Hillman, C.H., Kamijo, K. y Scudder, M. (2011). A review of chronic and acute physical activity participation on neuroelectric measures of brain health and cognition during childhood. Preventive Medicine, 52, S21-S28.

Hillman, C.H., Pontifex, M.B., Raine, L.B., Castelli, D.M., Hall, E.E. y Kramer, A.F. (2009). The effect of acute treadmill walking on cognitive control of academic achievement in preadolescent children. Neuroscience, 159(3), 1044-1054.

Hillman, C.H., Snook, E.M. y Jerome, G.J. (2003). Acute cardiovascular exercise and executive control function. International Journal of Psychophysiology, 48(3), 307-314.

Jones, S. y Drust, B. (2007). Physiological and technical demands of $4 \mathrm{v} 4$ and 8 v 8 games in elite youth soccer players. Kinesiology, 39(2), 150-156.

Katis, A. y Kellis, E. (2009). Effects of small-sided games on physical conditioning and performance in yough soccer players. Journal of Sport Science and Medicine, 8(3), 374-380.

Kempermann, G., Fabel, K., Ehninger, D., Babu, H., Leal-Galicia, P. Garthe, A. y Wolf, S.A. (2010). Why and how physical activity promotes experience-induced brain plasticity. Frontiers in Neuroscience, 4(189), 1-9.

Kramer, A.F. y Erickson, K.I. (2007). Capitalizing on cortical plasticity: influence of physical activity on cognition and brain function. Trends in Cognitive Sciences, 11(8), 342-348.

Kubesch, S., Walk, S., Spitzer, M., Kammer, T., Lainburg, A., Heim, R. y Hille, K. (2009). A 30-minute physical education program improves students' executive attention. Mind, Brain, and Education, 3(4), 235-242.

Lezak, M.D. (2004). Neuropsychological assessment. New York: Oxford University Press.

Manga, D. y Ramos, F. (2011). El legado de Luria y la neuropsicología escolar. Psychology, Society, \& Education, 3(1), 1-13.

Moral-García, J.E., Redecillas-Peiró, M.T. y Martínez-López, E.J. (2012) Hábitos sedentarios de los adolescentes andaluces. Journal of Sport and Health Research, 4(1), 67-82.

Mota, J., Santos, R.M., Silva, P., Aires, L., Martins, C. y Vale, S. (2012). Associations between self-rated health with cardiorespiratory fitness and obesity status among adolescent girls. Journal of physical activity \& health, 9(3), 378-381.

O'Hare, E.D. y Sowell, E.R. (2008). Imaging developmental changes in gray and white matter in the human brain. En C.A. Nelson y M. Luciana (Eds.), Handbook of Developmental Cognitive Neuroscience (pp. 23-38). Cambridge, MA: MIT Press.

O’Leary, K.C., Pontifex, M.B., Scudder, M.R., Brown, M.L. y Hillman, C.H. (2011). The effects of single bouts of aerobic exercise, exergaming, and videogame play on cognitive control. Clinical Neurophysiology, 122, 1518 1525 .

Padilla-Moledo, C., Castro-Piñero, J., Ortega, F.B., Mora, J., Márquez, S., Sjöström, M. y Ruiz, J.R. (2012). Positive health, cardiorespiratory fitness and fatness in children and adolescents. The European Journal of Public Health, 22(1), 52-56.

Powell, K.B. y Voeller, K.K.S. (2004). Prefrontal executive function syndromes in children. Journal of Child Neurology, 19(10), 785-797.

Ramos, P., Rivera, F., Moreno, C. y Jiménez-Iglesias, A. (2012). Análisis de clúster de la actividad física y las conductas sedentarias de los adolescentes españoles, correlación con la salud biopsicosocial. Revista de Psicología del Deporte, 21(1), 99-106.

Ratey, J. (2008). Spark. The revolutionary new science of exercise and the brain. New York: Little, Brown and Company.

Reitan, R. M. (1958). Validity of the Trail Making Test as an indication of organic brain damage. Perceptual and Motor Skills, 5, 271-276.

Reitan, R. M. (1992). Trail making test: Manual for administration and scoring. Tucson, AZ: Reitan Neuropsychology Laboratory.

Reitan, R.M. y Wolfson, D. (1985). The Halstead-Reitan Neuropsycholgical Test Battery: Therapy and clinical interpretation. Tucson, AZ: Neuropsychological Press.

Rigoli, D., Piek, J.P., Kane, R. y Oosterlaan, J. (2012). An examination of the relationship between motor coordination and executive functions in adolescents. Developmental Medicine and Child Neurology, 54(11), 1025-1231. 
Sampaio, J., Abrantes, C. y Leite, N. (2009). Power, heart rate and perceived responses to $3 \times 3$ and $4 \times 4$ basketball small-sided games. Revista de Psicología del Deporte, 18 (suppl.), 463-467.

Shallice, T. (1994). Multiple levels of control processes. En C. Umilta y M. Moscovitch (Eds.), Attention and performance XV (pp. 395-420). Cambridge, MA: MIT Press.

Soprano, A.M. (2003). Evaluación de las funciones ejecutivas. Revista de Neurología, 37(1), 44-50.

Stelzer, F., Mazzoni, C.C. y Cervigni, M.A. (2014). Cognitive models of executive functions development. Methodological limitations and theoretical challenges. Anales de Psicología, 30(1), 329-336.

Stroop, J.R. (1935). Studies of interference in serial verbal reactions. Journal of Experimental Psychology, 18, 643-662.

Stroth, S., Kubesch, S., Dieterle, K., Ruschsow, M., Heim, R. y Kiefer, M. (2009). Physical fitness, but no acute exercise modulates event-related potential indices for executive control in healthy adolescents. Brain Research, 1269, 114-124.

Swanson, H.L. (1996). Individual and age-related differences in children's working memory. Memory and Cognition, 24(1), 70-82.

Tombaugh, T. N. (2004). Trail Making Test A and B: Normative data stratified by age and education. Archives of Clinical Neuropsychology, 19(2), 203214.

Tomporowski, P.D., Davis, C.L., Miller, P.H. y Naglieri, J.A. (2008). Exercise and Children's Intelligence, Cognition, and Academic Achievement. Educational Psychology Review, 20(2), 111-131.
Tomporowski, P.D., Lambourne, K. y Okumura, M.S. (2011). Physical activity interventions and children's mental function: An introduction and overview. Preventive Medicine, 52(Suppl 1), S3-S9.

Verdejo-García, A. y Bechara, A. (2010). Neuropsicología de las funciones ejecutivas. Psicothema, 22(2), 227-235.

Verdejo-García, A., Pérez-Expósito, M., Schmidt-Río-Valle, J., FernándezSerrano, M.J., Cruz, F., Pérez-García, M., ... Campoy, C. (2010). Selective Alterations Within Executive Functions in Adolescents With Excess Weight. Obesity, 18(8), 1572-1578.

Wechsler, D. (2003). Wechsler Intelligence Scale for Children - Fourth Edition: Technical and interpretative manual. San Antonio, TX: Psychological Corporation.

Wechsler, D. (2005). Manual de aplicación y corrección del WISC-IV. Madrid: TEA Ediciones, S.A.

Wu, C.T., Pontifex, M.B., Raine, L.B., Chaddock, L., Voss, M.W., Kramer, A.F. y Hillman, C.H. (2011). Aerobic fitness and response variability in preadolescent children performing a cognitive control task. Neuropsychology, 25(3), 333-341.

Zelazo, P.D. y Carlson, S. (2012). Hot and Cool Executive Function in Childhood and Adolescence: Development and Plasticity. Child Development Perspectives, 6 (4) 354-360.

(Articulo recibido: 20-03-2013; revisado: 30-07-2013; aceptado: 13-09-2014) 